\title{
A SIMPLE CLOSED FORM FOR TRIANGULAR MATRIX POWERS*
}

\author{
WALTER SHUR
}

Abstract. Given a $k \times k$ triangular matrix $M=\left[m_{i, j}\right]$ with unique diagonal elements, a simple recursive formula is used to define a set of $\left(\begin{array}{c}k+2 \\ 3\end{array}\right)$ power factors, $p_{i, j, s}$, which are independent of the power to which the matrix is raised. Then for any power of $\mathrm{M}$, negative, zero or positive (positive only, if the matrix is singular), the $(i, j)$-th element of $M^{n}$ is given by a linear combination of power factors and powers of diagonal elements, namely ${ }_{n} m_{i, j}=\sum_{s=i}^{j} p_{i, j, s} m_{s, s}^{n-1}$.

Key words. Matrix, Triangular, Powers, Closed form.

AMS subject classifications. 15A99.

1. The algorithm. Huang [1] gives an algorithm for computing the powers of a triangular matrix where the diagonal elements are unique. However, in contrast to Huang's algorithm, the method presented here has the unique advantage of producing the result in closed form, which shows explicitly how the behavior of any element of the matrix varies with varying powers of the matrix. Also, the closed form may be helpful in discovering or proving some other bound or relationship.

Definition 1.1. Let $M=\left[m_{i, j}\right]$ be a $k \times k$ upper triangular matrix with unique diagonal elements. We define the power factors of $M, p_{i, j, s}$, recursively on the index $j$, as follows:

$$
\begin{array}{rlr}
p_{i, j, s}=\frac{\sum_{t=s}^{j-1} p_{i, t, s} m_{t, j}}{m_{s, s}-m_{j, j}} & i \leq s<j \leq k, \\
p_{i, j, s}=0 & s<i, s>j, \\
p_{i, j, j}=m_{i, j}-\sum_{t=i}^{j-1} p_{i, j, t} & i<j \leq k, \\
p_{j, j, j}=m_{j, j} . &
\end{array}
$$

\footnotetext{
*Received by the editors on March 29, 2011. Accepted for publication on August 25, 2011. Handling Editor: Bryan L. Shader.

$\dagger 20$ Speyside Circle, Pittsboro, NC 27312 (wrshur@gmail.com).
} 
LEMMA 1.2. If ${ }_{r} m_{i, j}=\sum_{s=i}^{j} p_{i, j, s} m_{s, s}^{r-1}$, then ${ }_{r+1} m_{i, j}=\sum_{s=i}^{j} p_{i, j, s} m_{s, s}^{r}$.

Proof. Since $M^{r+1}=M^{r} \cdot M,{ }_{r} m_{i, t}=0$, if $t<i$, and $m_{t, j}=0$ if $t>j$, we have

$$
{ }_{r+1} m_{i, j}=\sum_{t=i}^{j}{ }_{r} m_{i, t} m_{t, j} \text {. }
$$

Assuming that ${ }_{r} m_{i, j}=\sum_{s=i}^{j} p_{i, j, s} m_{s, s}^{r-1}$, (taking $0^{0}$ as 1 if $\mathrm{M}$ is singular), and noting that $p_{i, t, s}=0$ if $s>t$, we have

$$
{ }_{r+1} m_{i, j}=\sum_{t=i}^{j} m_{t, j} \sum_{s=i}^{t} p_{i, t, s} m_{s, s}^{r-1}=\sum_{s=i}^{j} m_{s, s}^{r-1} \sum_{t=s}^{j} m_{t, j} p_{i, t, s} .
$$

Splitting the rightmost sum, and substituting from Definition 1.1,

$\sum_{t=s}^{j} m_{t, j} p_{i, t, s}=\sum_{t=s}^{j-1} m_{t, j} p_{i, t, s}+m_{j, j} p_{i, j, s}=p_{i, j, s}\left(m_{s, s}-m_{j, j}\right)+m_{j, j} p_{i, j, s}=p_{i, j, s} m_{s, s}$.

Hence,

$$
r+1 m_{i, j}=\sum_{s=i}^{j} p_{i, j, s} m_{s, s}^{r}
$$

THEOREM 1.3. If $M=\left[m_{i, j}\right]$ is a non-singular upper triangular matrix with unique diagonal elements, and ${ }_{n} m_{i, j}$ is the $(i, j)$-th element of $M^{n}$, then

$$
{ }_{n} m_{i, j}=\sum_{s=i}^{j} p_{i, j, s} m_{s, s}^{n-1}
$$

for all integral values of $n$, negative, positive or zero. If $M$ is singular, the equation holds if $n \geq 1$ and $0^{0}$ is taken as 1 .

Proof. From the last two equations in Definition 1, we see that the theorem is true for $n=1$. From Lemma 1.2 , it is clearly true for all values of $n>1$.

If the elements of $M^{n-1}$ are given by ${ }_{n-1} m_{i, j}=\sum_{s=i}^{j} p_{i, j, s} m_{s, s}^{n-2}$, we know from Lemma 1.2 that $M^{n-1} * M=M^{n}$. And since $M^{n-1}$ is unique if $M^{-1}$ exists, the theorem is true for $n-1$, and hence true for all values of $n<1$.

Note that to obtain the powers of a lower triangular matrix, simply transpose to obtain an upper triangular matrix, apply the method described above, and transpose back. Alternatively, Definition 1.2 and Theorem 1.3 could be modified to apply directly to the lower triangular matrix. 
2. Illustration. Let

$$
M=\left(\begin{array}{llll}
4 & 2 & 3 & 1 \\
0 & 2 & 3 & 4 \\
0 & 0 & 1 & 4 \\
0 & 0 & 0 & 3
\end{array}\right)
$$

Here is how the factors $p_{1, j, s}$ are calculated from Definition 1.1:

$$
\begin{aligned}
& p_{1,1,1}=4, \\
& p_{1,2,1}=\frac{2 \cdot 4}{4-2}=4, \\
& p_{1,3,1}=\frac{3 \cdot 4+3 \cdot 4}{4-1}=8, \\
& p_{1,4,1}=\frac{1 \cdot 4+4 \cdot 4+4 \cdot 8}{4-3}=52, \\
& p_{1,2,2}=2-4=-2, \\
& p_{1,3,2}=\frac{3 \cdot(-2)}{2-1}=-6, \\
& p_{1,4,2}=\frac{4 \cdot(-2)+4 \cdot(-6)}{2-3}=32, \\
& p_{1,3,3}=3-8-(-6)=1, \\
& p_{1,4,3}=\frac{4 \cdot 1}{1-3}=-2, \\
& p_{1,4,4}=1-52-32-(-2)=-81 .
\end{aligned}
$$

Then we have from Theorem 1.3, for example,

$$
{ }_{n} m_{1,4}=52 \cdot 4^{n-1}+32 \cdot 2^{n-1}-2 \cdot 1^{n-1}-81 \cdot 3^{n-1} .
$$

3. Closing comments. Huang [1] states that the maximum number of multiplications and divisions required by his method to determine all of the elements of an $n \times n$ matrix raised to the $k$-th power is $n(k-1)+2 \sum_{p=1}^{n-1} p(n-p)$ (which is equal to $\left.2\left(\begin{array}{c}n+1 \\ 3\end{array}\right)+n(k-1)\right)$.

The comparable number for the method in this paper is $2\left(\begin{array}{c}n+2 \\ 3\end{array}\right)+n(k-3)$, which is of the same order of magnitude as the Huang number for large matrices [1]. But since the power factors use only the elements in the original matrix, once they are calculated, the elements in any power of that matrix can be computed in any order and far more easily than by the Huang method [1]. 
It should be noted that Huang [1] gives a second algorithm which can be used where the diagonal elements are not unique. Because that algorithm does not employ any division (only multiplication and addition), it cannot serve as a basis for an algorithm of the type presented in this paper.

\section{REFERENCES}

[1] C.P. Huang. An efficient algorithm for computing powers of triangular matrices. ACM 'r/8 Proceedings of the 1978 Annual Conference, 2:954-957, 1978. 